\title{
THE EFFECTS OF RADIATION, INCLINATION AND INSULATION OPACITY ON THE CRITICAL RADIUS FOR RADIAL HEAT TRANSFER
}

\author{
I. S. HABIB \\ Department of Mechanical Engineering, University of Michigan-Dearborn, \\ Dearborn, MI 48128, U.S.A.
}

(Received 6 Octaber 1981 and in final form 12 February 1982)

\section{NOMENCLATURE}

$a_{r}, \quad$ Rosseland mean absorption coefficient;

$C$, defined by equation (8);

$F$, geometric factor for surface radiation taken as equal to 1 ;

$g$, gravity

$G$, Grashof number, $g \beta\left(t_{0}-t_{\alpha_{s}}\right) x^{3} / v^{2}$;

$h$, natural convection heat transfer coefficient

$k, K, \quad$ thermal conductivity of insulation;

$k_{\mathrm{f}}, \quad$ thermal conductivity of air ;

$n$, index of refraction of insulation, defined by equation (9);

Pr, Prandtl number :

$q$, heat flux;

$O, \quad$ total heat transfer $\left[\mathrm{W} \mathrm{m}^{-1}\right]$

$r, R, \quad$ radial distance from center of cylinder;

$\boldsymbol{R}_{0}, \quad$ exterior radius for an insulating cylinder;

$T, \quad$ temperature;

$x$, distance along cylinder.

\section{Greek symbols}

$\beta$, volume coefficient of expansion;

$G$ surface emissivity of insulation;

$\theta, \quad$ angle of inclination from the vertical;

$\kappa$, absorption coefficient of insulating layer;

$v$, kinematic viscosity;

$\sigma, \quad$ Stefan-Boltzmann constant

\section{Subscripts}

$\begin{array}{ll}\mathrm{c}, & \text { convective; } \\ \mathrm{i}, \mathrm{I}, & \text { interior surface of insulation; } \\ \mathbf{k}, & \text { conductive; } \\ \mathbf{r}, & \text { radiativc; } \\ 0, & \text { exterior surface of insulation; } \\ r_{0}^{-}, & \text {approaching exterior surface from within; } \\ r_{0}^{+}, & \text {approaching exterior surface from surroundings; } \\ x, & \text { local distance along cylinder; } \\ x, & \text { ambient or surroundings. }\end{array}$

\section{INTRODUCTION}

THE ANALYSIS presented in the literature [1-3] on the subject of critical radius of insulation treated effects and corrections such as surface radiation and a variable heat transfer coefficient. Radiation and convection were considered to be dependent on the outside radius and the exterior surface temperature. It appears that all the work cited on the subject focuses on a horizontal slender cylinder. Hence, under such constraints, the analysis yields a value or an expression for the critical radius which is uniform along the length of the cylinder. When the orientation of the cylinder is taken into consideration, a single critical radius is no longer valid but rather a local critical radius is more significant due to the variation of the convection heat transfer coefficient along the length of the cylinder. Accordingly, coating the cylinder with a uniform layer of insulation can result in an increase in the heat transfer rate in one portion of the cylinder and a decrease in another.
The aim of this communication is (i) to re-examine the effect of a variable heat transfer coefficient on the critical radius when the angle of inclination of the cylinder is included as an additional parameter, (ii) to assess the effect of the opacity of insulation on the critical radius and on the heat transfer rate when the insulation is semi-transparent with an absorption coefficient $\boldsymbol{k}$.

\section{ANALYSIS}

The model chosen for this analysis is shown in Fig. 1. It is a slender cylinder of length $L$ oriented arbitrarily at an angle $\theta$ from the vertical. The cylinder is coated with a layer of insulating material having a constant temperature on the inside while the exterior surface temperature, $T_{0}$, is considered to be dependent upon $r_{0}, \theta$, the distance $x$ along the cylinder and the insulation absorption coefficient $\kappa$. The dependency on $r_{0}, \theta$ and $x$ stems from a variable natural convection coefficient while the dependency on $\kappa$ arises in considering a semi-transparent insulating layer. The thermal conductivity and absorption coefficient of the layer are taken as constant. The appearance of an axial variation in the exterior temperature of the insulation does yield axial conduction and radiation terms. However, such terms are considered insignificant relative to the radial rates of heat transfer.

The problem is first formulated in a general way and subsequently reduced to the common case of opaque insulation. When a semi-transparent layer is considered, the energy equation within the layer takes the following form:

$$
\frac{1}{r} \frac{\mathrm{d}}{\mathrm{d} r}\left[r\left(q_{\mathrm{r}}+q_{\mathrm{k}}\right)\right]=0 .
$$

Introducing the expression for the conductive heat flux $q_{\mathrm{k}}$ in equation (1) and integrating between the inner radius $r_{i}$ (where $T=T_{\mathrm{j}}$ ) to any arbitrary radius yields the following nonlinear integral equation for the temperature distribution within the layer (see ref. [4] for the expression of $q_{\mathrm{r}}$ ):

$$
T-T_{\mathrm{i}}=\int_{r_{\mathrm{i}}}^{r} \frac{q_{\mathrm{r}} \mathrm{d} r}{k}-\frac{C_{1}}{k} \ln \left(\frac{r}{\boldsymbol{r}_{\mathrm{i}}}\right)
$$

where

$$
C_{1}=\frac{1}{\ln \left(r_{0} / r_{\mathrm{i}}\right)}\left[k\left(T_{\mathrm{i}}-T_{0}\right)+\int_{r_{\mathrm{i}}}^{r_{0}} q_{\mathrm{r}} \mathrm{d} r\right]
$$

and

$$
\left[-k \frac{\mathrm{d} T}{\mathrm{~d} r}+q_{\mathrm{r}}\right]_{r=r_{0}^{-}}=\left[h\left(T_{0}-T_{\infty}\right)+q_{\mathrm{r}}\right]_{r=r_{0}^{+}} .
$$

Equation (4) represents the energy balance at the exterior surface of the insulating layer. For an opaque layer, there is no radiative contribution within the layer so that $q_{\mathrm{r}}$ in equations (2) and (3) and $\left(q_{\mathrm{r}}\right)_{r^{-}}$in equation (4) are zero. $\left(q_{\mathrm{r}}\right)_{r_{0}}$ then takes the usual form of the surface radiation term. Hence, for this opaque case equation (4) becomes 


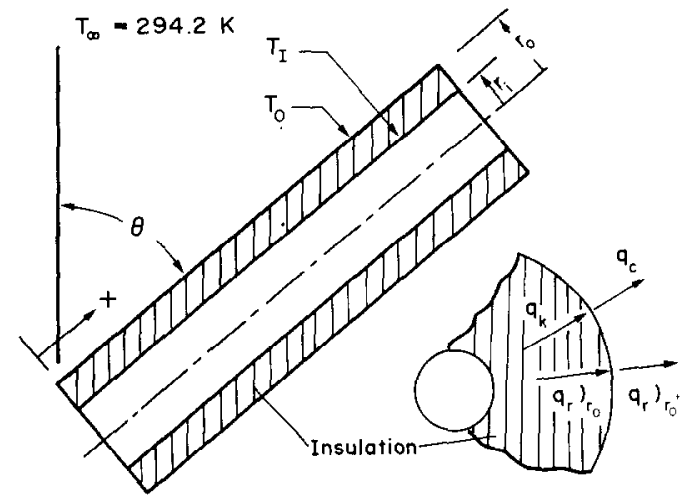

FiG. 1. The model chosen for this study.

$$
-k \frac{\mathrm{d} T}{\mathrm{~d} r}=h\left(T_{0}-T_{\alpha}\right)+q_{\mathrm{r}}
$$

or

$$
\frac{k\left(T_{i}-T_{0}\right)}{\ln \left(r_{0} / r_{i}\right)}=h r_{0}\left(T_{0}-T_{x}\right)+r_{0} \varepsilon F \sigma\left(T_{0}^{4}-T_{x}^{4}\right) .
$$

The existence of relationships for $h$ in the literature compatable with the present model are quite limited as the exterior surface is neither exposed to a constant temperature nor to a uniform heat flux. In view of such limitations, and because the concept of a local critical radius can be illustrated by any expression representing a local natural convection coefficient, the following relationship presented in ref. [5] was used:

$$
\frac{h_{x} x}{k_{\mathrm{f}}}=C\left(G r_{x} \operatorname{Pr}\right)^{n} \quad G r_{x} \operatorname{Pr} 1.48 \times 10^{8}
$$

with

$$
\begin{aligned}
& C=0.545-0.387(\sin \theta)^{1.462} \\
& n=1 / 4+\left(\frac{1}{12}\right)(\sin \theta)^{1.75} .
\end{aligned}
$$

\section{SOLUTION AND RESULTS}

As equations (2) - (7) do not possess analytical closed form solutions for the critical radius, a numerical iterative approach was used. For the case of opaque insulation, the solution is started by an assumed value for the exterior surface temperature, $T_{0} \cdot h_{x}$ is then calculated from equation (7). A new

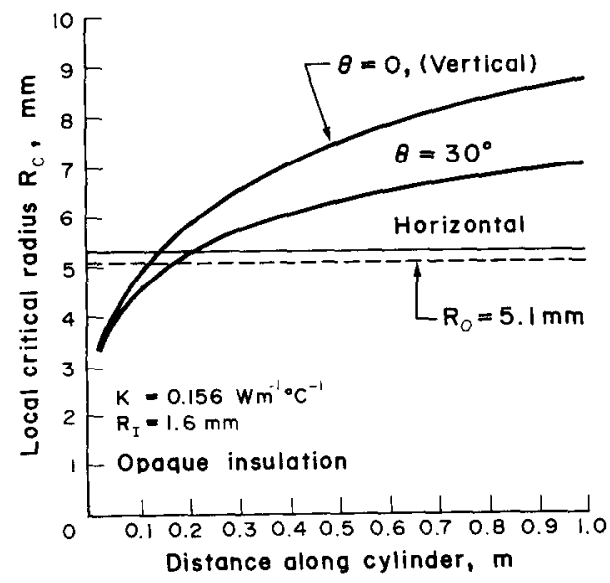

FIG. 2. Local critical radius along the cylinder-no radiation.

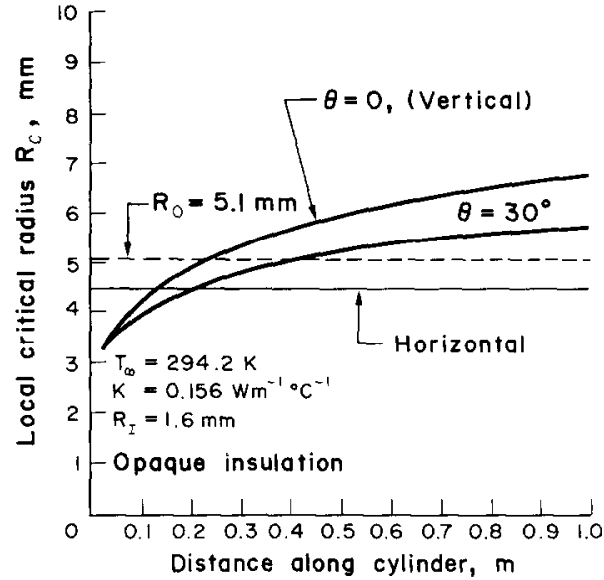

FIG. 3. Local critical radius along the cylinder-with surface radiation.

$T_{0}$ is subsequently obtained from equation (6) which is used again in equation (7) to give a new value for $h_{x}$. The procedure is repeated until convergence to a correct value for $T_{0}$ is achieved. The $T_{0}$ thus found is used to calculate the local radial heat transfer rate from the cylinder expressed by equation (6). For the semi-transparent insulation, an assumed profile for $T(r)$ is initially used in equation (3) and subsequently used also in equation (2) to generate $T(r)$ including $T_{0}$. The resulting $T(r)$ is used in equation (3) to calculate a new $C_{1}$ and hence a new $T(r)$ from equation (2). Repeating such iterations yielded a $T_{0}$ which was used to calculate $h_{x}$ from equation (7) and ultimately was used in equation (4) to calculate the radial heat transfer rate. The ambient temperature is chosen at $294.2 \mathrm{~K}$.

Figures 2 and 3 show the local critical radius, $r_{\mathrm{c}}$, along the cylinder for vertical, horizontal, and $30^{\circ}$ orientation when the insulation is opaque. A representative value (used in ref. [1]) for the thermal conductivity of insulation equal to $0.156 \mathrm{~W}$ $\mathrm{m}^{-1} \mathrm{C}^{-1}$ was used. The radius $r_{\mathrm{i}}$ of the bare cylinder was fixed at $1.6 \mathrm{~mm}$ with the temperature at this location maintained at $338.7 \mathrm{~K}$. Figure 2 , in which surface radiation was not included, shows a constant critical radius, as expected, for the horizontal cylinder as $h_{x}$ is invariant with $x$. Also shown in this figure are the results for a vertical and an inclined cylinder indicating a substantial variation in the values of the critical radius along the cylinder. When $r_{\mathrm{c}}$ is less than $r_{0}$, an

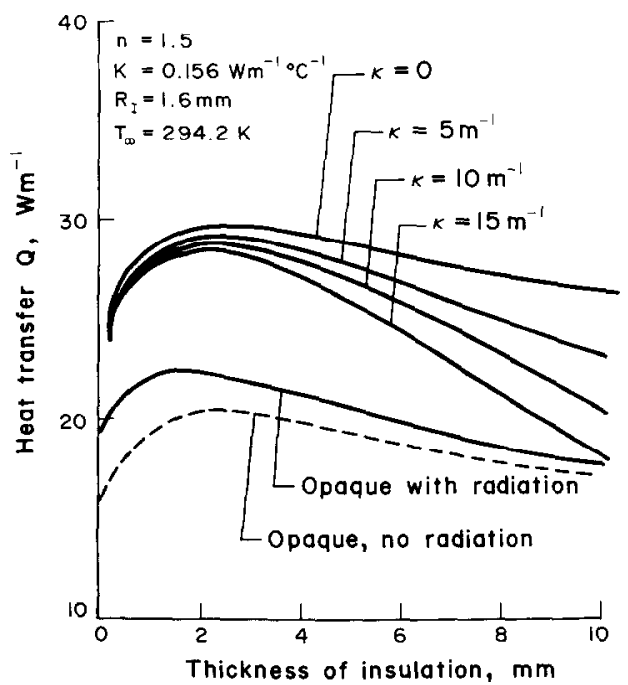

FIG. 4. Effect of insulation opacity on heat transfer. 
augmentation in the exterior radius $r_{0}$ (increasing the thickness of insulation) results in a reduction in the heat transfer rate in that segment of the cylinder. In the region where $r_{\mathrm{c}}$ is larger than $r_{0}$, an augmentation in thickness yields an increase in heat transfer rate there. Similar results are shown in Fig. 3 in which surface radiation is accounted for. With surface radiation the critical radius is expressed as $k /(h+$ ${ }_{4} F \sigma T_{0}^{3}$ ) where in the present analysis $\varepsilon$ and $F$ are taken as unity. In this case the critical radius follows a similar trend to that in Fig. 2 but consistently lower. For horizontal cylinders, the present results are in a slight disagreement with the results of ref. [1] due to the difference in the relationships used for the natural convection heat transfer coefficient.

Figure 4 shows the effect of thickness and opacity of a semitransparent insulation on the heat transfer rate from a horizontal cylinder. A horizontal case is chosen in order to isolate these effects from effects of inclination. The high rate of heat transfer exhibited for a zero absorption coefficient, $\kappa=$ 0 , is due to an additional factor, namely radiation leaving the inner surface of insulation at $r_{\mathrm{i}}$ with $T_{\mathrm{i}}$ directly to the exterior ambient in addition to the interior conduction and exterior convection. Increasing the opacity (increasing $\kappa$ and $r_{0}$ ) shifts the rate of heat transfer towards the opaque case, and insulation in this case plays the role of a radiation shield. As the optical thickness $\left(r_{0} \kappa\right)$ becomes very large, the insulation becomes essentially opaque and radiation becomes a surface phenomenon. This behavior can easily be verified by looking at the expression for the radiative heat flux $\left.q_{r}\right)_{r^{-}}$in the optically thick limit with black boundaries and temperature jump boundary conditions given as [6]

$$
\left[q_{\mathrm{r}}\right]_{r=r_{0}^{-}}=\frac{2 \pi r_{\mathrm{i}} \sigma\left[T_{\mathrm{i}}^{4}-T_{0}^{4}\right]}{\frac{3}{8}\left[2 a_{\mathrm{r}} r_{\mathrm{i}} \ln r_{\mathrm{o}} / r_{\mathrm{i}}+\frac{1-\left(r_{\mathrm{i}} / r_{0}\right)^{2}}{2 a_{\mathrm{r}} r_{\mathrm{i}}}\right]+\frac{1}{2}+r_{\mathrm{i}} / r_{0}}
$$

The value of this expression progressively decreases as $a_{\mathrm{r}}$ increases, and it approaches zero as $a_{\mathrm{r}}$ approaches infinity, thus yielding equation (6). Of interest is the location of the maximum value of heat transfer of the curves as they represent the position of the critical radius. The opaque insulation with surface radiation exhibits the lowest value for $\boldsymbol{r}_{\mathrm{c}}$. $A$ reduction in the opacity shifts the peaks to the right and hence to a larger value for $r_{\mathrm{c}}$. This shift can be interpreted as a result of providing an effective $K$, comprising radiation and conduction, and hence decreasing the value of the internal resistance to heat transfer. Finally, it can be stressed that in insulating slender cylinders arbitrarily oriented, a need arises in having the thickness of insulation compatable with the orientation as well as with the opacity of insulation. It is important to point out also that the idea of a local critical radius can be extended to a circumferentially variable heat transfer coefficient for the case of a horizontal cylinder. Extreme caution, however, should be exercised if the convection coefficient is considered to vary both axially and circumferentially over the cylinder due to the scatter of the existing data and the disagreement among the relationships recommended in the literature for natural convection from inclined surfaces.

\section{REFERENCES}

1. L. D. Simmons, Critical thickness of insulation accounting for variable convection coefficient and radiation loss, Trans. Am. Soc. Mech. Engrs, Series C, J. Heat Transfer 90, 150-152 (1976)

2. E. M. Sparrow, Re-examination and correction of the critical radius for radial heat conduction, A.I.Ch.E.J/ 16, 149 (1970).

3. T. H. Kuehn, Radial heat transfer and critical Biot number with radiation, uniform surface heat generation and curvature effects in convection, Trans. Am. Soc. Mech. Engrs, Series C, J. Heat Transfer 100, 374-376 (1978).

4. I. S. Habib, Solidification of a semi-transparent cylindrical medium by conduction and radiation, Trans. Am. Soc. Mech. Engrs, Series C, J. Heat Transfer 96, 37-41 (1973).

5. M. Al-Arabi and Y. K. Salman, Laminar natural convection heat transfer from an inclined cylinder, Int. $J$. Heat Mass Transfer 23, 45-51 (1980).

6. R. Siegel and J. R. Howell, Thermal Radiation Heat Transfer, p. 513. McGraw-Hill, Hemisphere, New York (1981).

\title{
PERFORMANCE OF COUNTER CURRENT HEAT EXCHANGER WITH PERIODIC INLET TEMPERATURES
}

\author{
M. S. SODHA \\ Centre of Energy Studies, Indian Institute of Technology, New Delhi 110016, India
}

(Received 15 September 1981 and in final form 23 January 1982)

\section{NOMENCLATURE}

$A_{1}, A_{2}$,

$c$,

areas of cross section of fluid channels; mean specific heat of the heat exchanger mass

$c_{1}, c_{2}, \quad$ specific heats of fluids at constant pressure;

$C_{0}\left(\mu_{0}-1\right), C_{0}^{\prime}\left(\mu_{0}-1\right)$, constants of integration

$C_{1 m}\left(\mu_{0}-1\right), C_{2 m}\left(\mu_{0}-1\right)$, constants of integration;

$h$. heat transfer between the fluids per unit time per unit length per unit difference of temperature:

$m, \quad$ integer $(1-\infty)$;

$M_{1}, M_{2}$, heat exchanger mass per unit length, corresponding to the two channels;

$t$ time

$u_{1}, \quad h /\left(A_{1} \rho_{1} c_{1}+M_{1} c\right)$

$u_{2}, \quad h /\left(A_{2} \rho_{2} c_{2}+M_{2} c\right)$

$$
\begin{array}{ll}
v_{1}, & {\left[\frac{A_{1} \rho_{1} c_{1} v_{1}^{\prime}}{A_{1} \rho_{1} c_{1}+M_{1} r}\right] ;} \\
v_{2}, & {\left[\frac{A_{2} \rho_{2} c_{2} v_{2}^{\prime}}{A_{2} \rho_{2} c_{2}+M_{2} c}\right] ;} \\
v_{1}^{\prime}-v_{2}^{\prime}, & \begin{array}{l}
\text { fluid flow velocities; } \\
x,
\end{array} \\
& \begin{array}{l}
\text { distance along heat exchanger, } \\
\left(u_{2} / v_{2}\right)-\left(u_{1} / v_{1}\right) .
\end{array}
\end{array}
$$

Greek symbols

$$
\begin{array}{ll}
\alpha_{m}^{+}, \alpha_{m}^{-}, & \text {complex roots of equation }(8) ; \\
\beta_{m}, & {\left[\left(u_{2}+\text { im }\right) / v_{2}+\left(\alpha_{m} u_{1} / v_{1}\right)\right] ;} \\
\mu_{0}, & =\left(u_{2} / v_{2}\right) /\left(u_{1} / v_{1}\right) ; \\
\rho_{1}, \rho_{2}, & \text { density of fluids; } \\
\omega, & 2 \pi / \text { period. }
\end{array}
$$

